\title{
Alternatively spliced tissue factor levels are elevated in the plasma of patients with chronic liver diseases
}

\author{
Nathalie I. Caversaccio a,b,d, Maria D. Reina Caro ${ }^{a, d}$, Raja Prince ${ }^{a, d}$, Martin Müller ${ }^{c, e}$, Clayton S. Lewis $^{f}$, \\ Vladimir Y. Bogdanov ${ }^{f}$, Jean-François Dufour ${ }^{\mathrm{b}, \mathrm{d}, \star}$ and Anne Angelillo-Scherrer ${ }^{\mathrm{a}, \mathrm{d}, \star}$
}

\begin{abstract}
Objectives In patients with chronic liver diseases, hypercoagulability can contribute to the progression of fibrosis and complications of cirrhosis. Tissue factor (TF) is a transmembrane glycoprotein that initiates the extrinsic pathway of blood coagulation. Recent investigations have established that TF is elevated in patients with pancreatic cancer, blood disorders, diabetes, and cardiovascular disease. Alternatively spliced tissue factor (asTF), a secreted form of TF, induces angiogenesis and exhibits low-level procoagulant activity. The aim of this study was to investigate whether the circulating levels of asTF are elevated in the plasma of patients with liver disease.

Materials and methods In a single-center study, we retrospectively analyzed asTF plasma levels in healthy participants and patients having stage FO-F3 liver fibrosis, liver cirrhosis, as well as hepatocellular carcinoma (HCC). AsTF plasma levels were measured using a sandwich enzyme-linked immunosorbent assay. Values were expressed as median with interquartile range (IQR).

Results The lowest median plasma asTF concentration (94 pg/ml, IQR: 33-275) was found in the healthy control group. The patients with low-grade liver fibrosis (FO-F1 group) displayed the highest median asTF concentration (404 pg/ml, IQR: 277-789). Significant differences between the asTF levels in the plasma of healthy participants and those in patients with grade F0-F1 fibrosis $(P<0.001)$, patients with grade F2-F3 fibrosis $(P=0.019)$, patients with cirrhosis $(P=0.004)$, and patients with HCC $(P<0.001)$ were found using a Wilcoxon rank-sum test. Treatment-naive patients with HCC had significantly higher asTF levels $(P=0.018)$ than those receiving treatment. AsTF levels were found to increase with worsening Child-Pugh scores and heightened liver disease activity.

Conclusion AsTF levels are elevated in patients with chronic liver diseases, which increase with worsening Child-Pugh scores and decrease following HCC therapy. Eur J Gastroenterol Hepatol 00:000-000

Copyright (? 2018 Wolters Kluwer Health, Inc. All rights reserved.
\end{abstract}

\section{Introduction}

The coagulation system participates in the progression of liver diseases. It has been recently recognized that the formation of microthrombi in the hepatic sinusoids and elevated plasma levels of von Willebrand factor are associated with more advanced liver disease [1-3]. The lack of von Willebrand factor reduces the progression of liver

European Journal of Gastroenterology \& Hepatology 2018, 00:000-000

Keywords: coagulation, fibrosis, liver, hepatic perfusion, tissue factor

aDepartment of Hematology and Central Hematology Laboratory, bUniversity Clinic for Visceral Surgery and Medicine, 'Department of Emergency Medicine, Inselspital, Bern University Hospital, dDepartment for BioMedical Research (DBMR), University of Bern, Bern, Switzerland, eInstitute of Health Economics and Clinical Epidemiology, University Hospital of Cologne, Cologne, Germany and

fDepartment of Internal Medicine, Division of Hematology/Oncology, University of Cincinnati College of Medicine, Cincinnati, Ohio, USA

Correspondence to Jean-François Dufour, MD, University Clinic for Visceral Surgery and Medicine, Inselspital, Bern, Switzerland, Freiburgstrasse, CH-3010

Bern, Switzerland

Tel: +4131632 26 95; fax: +413163297 65;

e-mail: jean-francois.dufour@insel.ch

*Jean-François Dufour and Anne Angelillo-Scherrer contributed equally to the writing of this article.

Received 15 March 2018 Accepted 27 July 2018

Supplemental Digital Content is available for this article. Direct URL citations appear in the printed text and are provided in the HTML and PDF versions of this article on the journal's website, www.eurojgh.com. fibrosis in mice through a reduction in collagen deposition [3]. Low levels of factor VII activating protease are associated with enhanced liver fibrosis in humans [4]. However, many aspects of the role of coagulation factors in hepatic pathophysiology remain to be investigated.

Tissue factor (TF) is a transmembrane glycoprotein that initiates the extrinsic pathway of blood coagulation by acting as a cellular receptor and cofactor for factor VII. TF is expressed in monocytes, fibroblasts, smooth muscle cells, cerebral cortex, renal glomeruli, cardiac myocytes, spleen trabeculae and capsule, gut mucosa, epidermis, bronchial mucosa, alveolar epithelial cells, and Schwann cells $[5,6]$. Hepatocytes also express TF but at low concentrations [5].

Circulating TF ('blood-borne' or TF in cell-free plasma) exists in two forms: full-length tissue factor (fTTF), encoded by 6 exons, and alternatively spliced tissue factor (asTF), with a deletion of exon 5 owing to alternative splicing of exon 4 directly to exon 6 ; this leads to a frame shift in exon 6 , yielding a transmembrane domain-lacking isoform that can be secreted $[7,8]$. Regulation of TF pre-mRNA splicing was shown to depend on SR proteins in monocytes and endothelial cells $[9,10]$. Additionally, it has been reported that TF gene expression is regulated by nuclear factor- $\mathrm{kB}$ [11].

Recent investigations have established that total circulating TF is elevated in patients with diverse conditions such as diabetes mellitus, myocardial infarction, unstable 
angina pectoris, sickle-cell disease, antiphospholipid antibody syndrome and pancreatic cancer [12-16]. These patients are at risk of developing thrombosis owing to higher activity of circulating TF $[12,16]$. In patients with cirrhosis, TF expression is increased in endothelial cells of splenic vein induced by interleukin-17A, an inflammatory cytokine which is elevated in liver cirrhosis [17]. Rautou et al. [18] have shown that hepatocyte TF contributes to the activation of coagulation in chronic liver disease.

AsTF induces angiogenesis by acting as an integrin ligand and can also promote coagulation in association with phospholipid surfaces [7,19]. AsTF has been reported to act on cancer cells by $\beta 1$ integrins and to induce cell proliferation, which contributes to breast and pancreatic ductal carcinoma growth $[20,21]$. Patients with pancreatic cancer have significantly higher plasma asTF concentrations than healthy participants, and high asTF levels in circulation are significantly associated with a lower likelihood of tumor resectability [22]. As recently published, targeting asTF can impede tumor growth in an orthotopic murine model of pancreatic ductal adenocarcinoma [23].

The aim of this study was to investigate whether the circulating levels of asTF are elevated in the plasma of patients with liver fibrosis, cirrhosis, and hepatocellular carcinoma (HCC).

\section{Materials and methods}

\section{Study design}

This observational, single-center study retrospectively analyzed data from patients with liver disease and healthy blood donors. Patients' plasma samples were collected between 2011 and 2017 at the Department of Visceral Surgery and Medicine, Bern University Hospital, Switzerland. Healthy participants' plasma samples were collected between January and March 2017 by voluntary blood donations. All patients and healthy volunteers signed an informed consent allowing use of plasma samples for research. The study was conducted with the approval of the Kantonale Ethikkommission Bern (Basec-Nr. 2016-00213).

\section{Data collection}

Patients had liver disease owing to hepatitis B virus (HBV) and hepatitis $\mathrm{C}$ virus $(\mathrm{HCV})$, alcoholic steatohepatitis (ASH), nonalcoholic steatohepatitis (NASH), hereditary hemochromatosis, $\alpha-1$-antitrypsin deficiency and cryptogenic liver disease. Transient elastography (TE) cutoffs have been used according to etiologies for staging liver fibrosis (EASL-ALEH Clinical Practice Guidelines) [24]. The determination of TE for the measurement of liver stiffness occurred no more than one month from the time point of plasma acquisition. Nonadvanced fibrosis (fibrosis stage 0-1) was defined on a cutoff of less than $6.0 \mathrm{kPa}$ in $\mathrm{HBV}$, less than $5.2 \mathrm{kPa}$ in $\mathrm{HCV}$, less than $7.0 \mathrm{kPa}$ in ASH/NASH, and less than $7.9 \mathrm{kPa}$ in hereditary hemochromatosis, $\alpha-1$-antitrypsin deficiency, and cryptogenic liver disease. Fibrosis stage $2-3$ was defined on a cutoff of $6.0-10.9 \mathrm{kPa}$ in $\mathrm{HBV}, 5.2-12.8 \mathrm{kPa}$ in $\mathrm{HCV}$, $7.0-10.2 \mathrm{kPa}$ in ASH/NASH, and $7.9-11.8 \mathrm{kPa}$ in hereditary hemochromatosis, $\alpha-1$-antitrypsin deficiency, and cryptogenic liver disease.

For the diagnosis of cirrhosis (fibrosis stage 4), cutoff of $11.0 \mathrm{kPa}$ in $\mathrm{HBV}, 12.9 \mathrm{kPa}$ in $\mathrm{HCV}, 10.3 \mathrm{kPa}$ in $\mathrm{ASH} /$
$\mathrm{NASH}$, and $11.9 \mathrm{kPa}$ in hereditary hemochromatosis, $\alpha$ 1-antitrypsin deficiency, and cryptogenic liver disease were used [24].

The HCC group comprised patients with tumors who had been diagnosed based on typical radiological criteria using computed tomography or MRI with contrast enhancement.

The control group comprised blood donors matched by sex and age. These participants were healthy because the Swiss Red Cross had deemed them eligible as blood donors after their usual checkup procedure, which includes screening for a number of organ dysfunctions, notably liver diseases. Plasma samples were provided by the Interregional Blood Transfusion Service of the Swiss Red Cross, Bern, Switzerland.

\section{Enzyme-linked immunosorbent assay}

The obtained samples were stored at $-80^{\circ} \mathrm{C}$ and gently thawed before testing. AsTF-specific sandwich enzymelinked immunosorbent assay was used to evaluate as TF protein levels in plasma samples [25]. In brief, plasma samples were added to microplate wells precoated with a capture antibody. A washing step was then performed to remove the unbound material. Afterwards, peroxidaseconjugated monoclonal antibody specific for human asTF was added to the wells. To remove the excess of anti-asTF antibody-peroxidase conjugate, another washing step was carried out. The action of the bound peroxidase was revealed by its reaction with tetramethylbenzidine. The reaction was stopped by sulfuric acid $\left(\mathrm{H}_{2} \mathrm{SO}_{4}\right)$, and the absorbance at $450 \mathrm{~nm}$ was immediately measured. The color intensity was directly proportional to the amount of asTF in the sample, derived using asTF protein standard curve. For the batch of prototype enzyme-linked immunosorbent assay sets used in this study (10 96-well kits), a consistently reliable sensitivity cutoff across the entire study was $500 \mathrm{pg} / \mathrm{ml}$ (Supplementary Table S1, Supplemental digital content 1, http://links.lww.com/EJGH/A330).

\section{Statistical analysis}

Stata 13.1 (StataCorp, College Station, Texas, USA) was used to perform the statistical analysis. Visually and statistically (Shapiro-Wilk test), asTF levels as well as their log-transformation were not normally distributed. Thus, asTF levels were reported as median with interquartile range (IQR), and nonparametric tests were used for group comparisons. The Kruskal-Wallis test with the Wilcoxon rank-sum test as a post-hoc test was performed to compare the distribution of continuous variables between the groups. The Spearman's correlation coefficient was calculated to test for a linear association between asTF values and different variables. $P$ values less than 0.05 were considered statistically significant.

\section{Results}

AsTF levels were measured in the plasma of 60 healthy participants, 48 patients having stage $0-1$ liver fibrosis (F0-F1 group), 33 patients with stage 2-3 liver fibrosis (F2-F3 group), 65 patients with liver cirrhosis (cirrhotic group), and 128 patients with HCC. The patients' major characteristics are shown in Table 1. 
The most common etiology of liver disease was ASH and NASH. The second largest group comprised patients with chronic viral infection, that is, hepatitis B and hepatitis C. A total of 37 patients with cryptogenic liver disease, hereditary hemochromatosis, and $\alpha$-1-antitrypsin deficiency were categorized into the mixed etiology group.

The median asTF concentrations for each patient group are shown in Table 2. The lowest median plasma asTF concentration (94 pg/ml, IQR: 33-275) was detected in the healthy control group. The patients with low-grade liver fibrosis (F0-F1 group) displayed the highest median asTF concentration (404 pg/ml, IQR: 277-789), followed by patients with HCC (392 pg/ml, IQR: 232-812). Median plasma asTF for patients with an advanced liver fibrosis (F2-F3 group) was 356 (IQR: 44-859) pg/ml. In patients with cirrhosis, median plasma asTF was 228 (IQR: 108-442) pg/ml. Correlation analysis is shown in Table 3.

Plasma asTF concentrations in healthy participants (94 pg/ml, IQR: 33-275) were significantly lower compared with all patients with chronic liver disease $(373 \mathrm{pg} /$ ml, IQR: 191-721) (Fig. 1).

Healthy participants were found to have significantly less circulating asTF than patients with grade F0-F1 fibrosis, grade F2-F3 fibrosis, cirrhosis, and HCC. Additionally, both patients with grade F0-F1 fibrosis and those with HCC were found to have significantly higher circulating asTF than patients with cirrhosis as determined by a Wilcoxon rank-sum test (Fig. 2).

There were no effects of sex, age and underlying liver disease (HBV, HCV, ASH/NASH, and other liver disease) on asTF levels (Table 3 and Supplementary Table S2, Supplemental digital content 1, http://links.lww.com/ EJGH/A330).
There was no correlation detectable between the grade of liver fibrosis (measured by TE) and asTF levels in patients with grade $0-4$ fibrosis $(r=-0.204, P=0.027)$ (Fig. 3) nor was there a correlation in untreated patients between the levels of circulating asTF and the size of HCC tumors measured radiologically $(r=0.030, P=0.865)$ (data not shown).

AsTF levels were not significantly affected by either HBV undertreatment $(P=0.398)$ or eradicated HCV $(P=0.076)$ or by active alcohol consumption $(P=0.069)$ in patients with ASH (data not shown). However, patients with HCC who benefited from therapy such as liver transplantation, curative resection, trans-arterial chemoembolization, radiofrequency ablation, selective internal radiation therapy, and systemic therapy with sorafenib displayed significantly lower plasma asTF concentrations than treatment-naive patients (Fig. 4). Additionally, asTF levels rose with increased disease severity in patients with cirrhosis, with patients scored as Child-Pugh $\mathrm{C}$ having higher levels than those scored as Child-Pugh A (Fig. 5). Although a similar trend in circulating asTF was observed when patients with HCC were stratified based on their Child-Pugh scores, this did not reach significance. However, when all patients receiving Child-Pugh scores were analyzed together, that is, those with cirrhosis and/or HCC, it was found that patients with Child-Pugh C score had significantly higher circulating asTF than those with either Child-Pugh B $(P=0.036)$ or Child-Pugh A $(P=0.009)$ score.

Finally, we compared patients with untreated HBV and $\mathrm{HCV}$, active alcohol consumption, and active NASH with alanine aminotransferase (ALT) more than $50 \mathrm{U} / \mathrm{I}$ (active liver disease, $n=40$ ) versus patients with untreated HBV and $\mathrm{HCV}$, active alcohol consumption, and active NASH

Table 1. Demographics and clinical characteristics of healthy participants and patients with F0-F3 fibrosis, cirrhosis, and hepatocellular carcinoma

\begin{tabular}{|c|c|c|c|c|c|}
\hline & $\begin{array}{l}\text { Healthy participants } \\
\qquad(n=60)\end{array}$ & $\begin{array}{l}\text { Patients with grade } 0-1 \\
\text { fibrosis }(n=48)\end{array}$ & $\begin{array}{l}\text { Patients with grade } 2-3 \\
\text { fibrosis }(n=33)\end{array}$ & $\begin{array}{l}\text { Patients with cirrhosis } \\
\qquad(n=65)\end{array}$ & $\begin{array}{l}\text { Patients with HCC } \\
\quad(n=128)\end{array}$ \\
\hline Age [median (IQR)] (years) & $58(57-60)$ & $53(45-61)$ & $56(42-60)$ & $58(50-62)$ & $66(59-74)$ \\
\hline \multicolumn{6}{|l|}{$\operatorname{Sex}[n(\%)]$} \\
\hline Male & 35 (58.3) & $28(58.3)$ & $23(69.7)$ & $46(70.8)$ & $111(86.7)$ \\
\hline Female & $25(41.7)$ & $20(41.7)$ & 10 (30.3) & $19(29.2)$ & 17 (13.3) \\
\hline \multicolumn{6}{|l|}{ Etiology $[n(\%)]$} \\
\hline HBV & $0(0.0)$ & $1(2.1)$ & $6(18.2)$ & $1(1.5)$ & $10(7.8)$ \\
\hline Undertreatment & $0(0.0)$ & $0(0.0)$ & 2 (33.3) & $0(0.0)$ & $8(80.0)$ \\
\hline $\mathrm{HCV}$ & $0(0.0)$ & $13(27.1)$ & $16(48.5)$ & $11(16.9)$ & $38(29.7)$ \\
\hline Eradicated & $0(0.0)$ & $2(15.4)$ & $7(43.8)$ & $0(0.0)$ & $20(52.6)$ \\
\hline ASH & $0(0.0)$ & $5(10.4)$ & $6(18.2)$ & $34(52.3)$ & $42(32.8)$ \\
\hline Active alcohol consumption & $0(0.0)$ & $4(12.9)$ & $5(45.5)$ & $12(27.9)$ & $14(25.0)$ \\
\hline NASH & $0(0.0)$ & $26(54.2)$ & $5(15.1)$ & 9 (13.9) & $14(10.9)$ \\
\hline Mixed liver disease & $0(0.0)$ & $3(6.2)$ & $0(0.0)$ & $10(15.4)$ & $24(18.8)$ \\
\hline HCC treatment [n (\%)] & - & - & - & - & $83(64.8)$ \\
\hline $\begin{array}{l}\text { Active liver disease (ALT > } 50 \mathrm{U} / \mathrm{l}) \\
\text { [n (\%)] }\end{array}$ & - & $20(45.5)$ & $9(27.3)$ & $11(16.9)$ & - \\
\hline AST [median (IOR)] (U/I) & - & $36(26-47)$ & $35(27-54)$ & $54(33-78)$ & $62(39-104)$ \\
\hline ALT [median (IOR)] (U/I) & - & $53(30-75)$ & $37(24-83)$ & $34(20-57)$ & $38(29-86)$ \\
\hline AFP [median (IOR)] (kU/l) & - & - & - & - & $11(5-110)$ \\
\hline Fibroscan [median (IQR)] (kPa) & - & $5.0(4.0-6.1)$ & $7.5(6.4-7.9)$ & $20.9(16-34.8)$ & - \\
\hline \multicolumn{6}{|l|}{ Child-Pugh score [n (\%)] } \\
\hline A & $0(0.0)$ & $0(0.0)$ & $0(0.0)$ & $22(41.5)$ & $83(64.8)$ \\
\hline B & $0(0.0)$ & $0(0.0)$ & $0(0.0)$ & $27(50.9)$ & $31(24.2)$ \\
\hline C & $0(0.0)$ & $0(0.0)$ & $0(0.0)$ & $4(7.6)$ & $4(3.1)$ \\
\hline MELD-score (6-40) [median (IOR)] & - & - & - & $11(9-13)$ & $8(7-11)$ \\
\hline $\begin{array}{l}\text { Tumor size in untreated patients } \\
\text { [median (IOR)] }\left(\mathrm{cm}^{2}\right)\end{array}$ & - & - & - & - & $31.3(8.8-79.5)$ \\
\hline
\end{tabular}

AFP, $\alpha$-fetoprotein; ALT, alanine aminotransferase; ASH, alcoholic steatohepatitis; HBV, hepatitis B virus; HCC, hepatocellular carcinoma; HCV, hepatitis C virus; IOR, interquartile range; MELD, model for end-stage liver disease; NASH, nonalcoholic steatohepatitis. 
Table 2. Plasma alternatively spliced tissue factor levels $(\mathrm{pg} / \mathrm{ml})$ in healthy participants and patients with fibrosis, cirrhosis, and hepatocellular carcinoma

\begin{tabular}{|c|c|c|c|c|c|}
\hline & $\begin{array}{l}\text { Healthy participants } \\
\qquad(n=60)\end{array}$ & $\begin{array}{l}\text { Patients with grade } 0-1 \\
\text { fibrosis }(n=48)\end{array}$ & $\begin{array}{l}\text { Patients with grade } 2-3 \\
\text { fibrosis }(n=33)\end{array}$ & $\begin{array}{l}\text { Patients with cirrhosis } \\
\qquad(n=65)\end{array}$ & $\begin{array}{c}\text { Patients with } \mathrm{HCC} \\
\quad(n=128)\end{array}$ \\
\hline \multicolumn{6}{|c|}{ asTF [median (IQR)] (pg/ml) } \\
\hline Males $(n=243)$ & $82(6-336)$ & 583 (303-981) & $270(9-859)$ & $213(108-409)$ & $410(233-908)$ \\
\hline Females $(n=91)$ & $135(41-242)$ & $349(222-421)$ & $515(275-1781)$ & 249 (104-468) & $364(170-559)$ \\
\hline Total $(n=332)$ & 94 (33-275) & 404 (277-789) & $356(44-859)$ & $228(108-442)$ & $392(232-812)$ \\
\hline
\end{tabular}

asTF, alternatively spliced tissue factor; HCC, hepatocellular carcinoma; IQR, interquartile range.

Table 3. Correlation analysis (Spearman's rank correlation coefficient) between plasma alternatively spliced tissue factor levels (pg/ml) and different clinical parameters

\begin{tabular}{lr}
\hline asTF and ... & $P$-value \\
\hline Age (patients with F0-F3 fibrosis, patients with cirrhosis and patients with HCC) $(n=274)$ & 0.032 \\
Sex (patients with F0-F3 fibrosis, patients with cirrhosis, and patients with HCC) $(n=274)$ & 0.051 \\
AFP (patients with cirrosis and patients with HCC) $(n=193)$ & 0.171 \\
AST (patients with FO-F3 fibrosis, patients with cirrhosis and patients with HCC) $(n=233)$ & 0.398 \\
ALT (patients with F0-F3 fibrosis, patients with cirrhosis, and patients with HCC) $(n=232)$ & 0.023 \\
MELD-score (patients with cirrhosis and patients with HCC) (n=181) & 0.008 \\
Tumor size (untreated patients with HCC) (n=36) & 0.001 \\
\end{tabular}

AFP, $\alpha$-fetoprotein; ALT, alanine aminotransferase; AST, aspartate aminotransferase; HCC, hepatocellular carcinoma; MELD, model for end-stage liver disease.

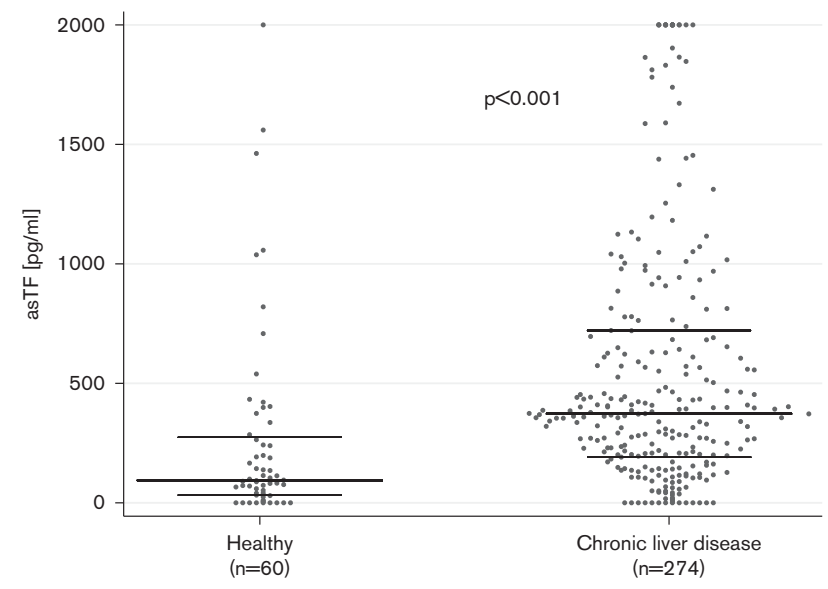

Fig. 1. Comparison of the distribution of plasma alternatively spliced tissue factor (asTF) levels in healthy participants $(n=60)$ and patients with chronic liver disease $(n=274)$. Thick bars indicate median asTF concentrations, and thin bars the interquartile range. For illustration purposes, asTF values more than $2000 \mathrm{pg} / \mathrm{ml}$ are shown as $2000 \mathrm{pg} / \mathrm{ml}$.

with ALT up to $50 \mathrm{U} / \mathrm{I}$ (no active liver disease, $n=44$ ). As a cutoff to define the activity of liver disease, we used ALT more than $50 \mathrm{U} / \mathrm{l}$. Patients with active liver disease showed significantly higher asTF levels $(583 \mathrm{pg} / \mathrm{ml}$, IQR: 221-1088) than patients with no active liver disease (371 pg/ml, IQR: 59-661) (Fig. 6).

\section{Discussion}

This is the first study that investigates the circulating levels of asTF in patients with liver fibrosis, cirrhosis, and HCC. Our data revealed that the asTF levels were elevated not only in patients with HCC but also in patients with cirrhosis and even in some patients with and without fibrosis. Interestingly, patients with no fibrosis or minimal fibrosis (F0-F1 group) showed significantly higher asTF levels than patients with cirrhosis. Because asTF expression levels usually follow flTF expression levels, this may be caused by

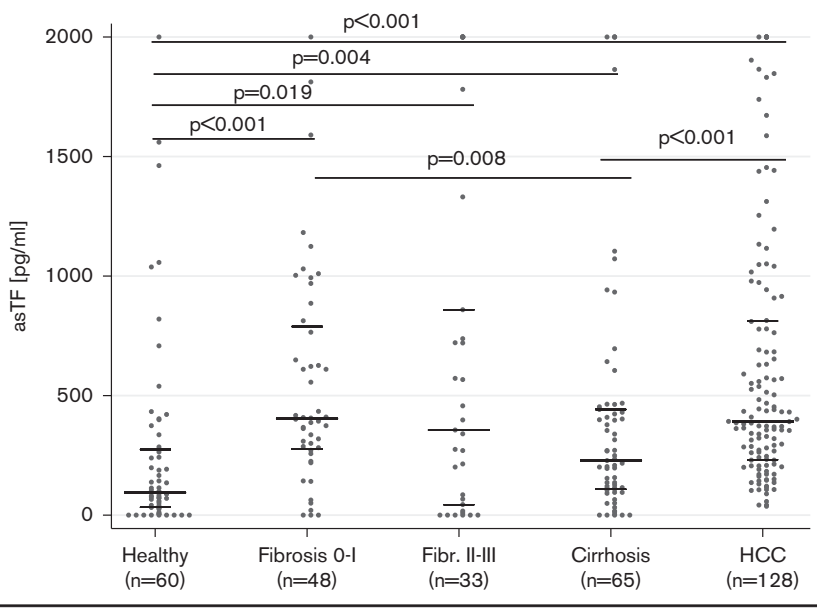

Fig. 2. Comparison of the distribution of plasma alternatively spliced tissue factor (asTF) levels in patients with fibrosis, patients with cirrhosis, and patients with hepatocellular carcinoma (HCC). Healthy participants $(n=60)$, patients with fibrosis $0-1(n=48)$, patients with fibrosis II-III $(n=33)$, patients with cirrhosis $(n=65)$, and patients with HCC $(n=128)$. Thick bars indicate median asTF concentrations, and thin bars the interquartile range. For illustration purposes, asTF values more than $2000 \mathrm{pg} / \mathrm{ml}$ are shown as $2000 \mathrm{pg} / \mathrm{ml}$.

an increased TF-dependent coagulation activity in a lowgrade fibrotic liver compared with a cirrhotic liver [26,27]. Thus, we propose that coagulation activation may occur early in the progress of liver disease and independently of fibrosis. Liver parenchymal cells synthesize most coagulation factors; however, in the case of hepatic insufficiency, their production is impaired. This leads to alterations in blood coagulation, with disruptions in the balance between procoagulation and anticoagulation factors [26].

An earlier study showed that asTF levels were positively associated with an increased thrombotic tendency in chronic hemodialysis patients [28]. The lack of a correlation between grade of liver fibrosis and plasma asTF levels may be owing to a contribution to the formation of microthrombi in hepatic sinusoids promoting liver disease progression rather than a direct involvement of asTF in the 


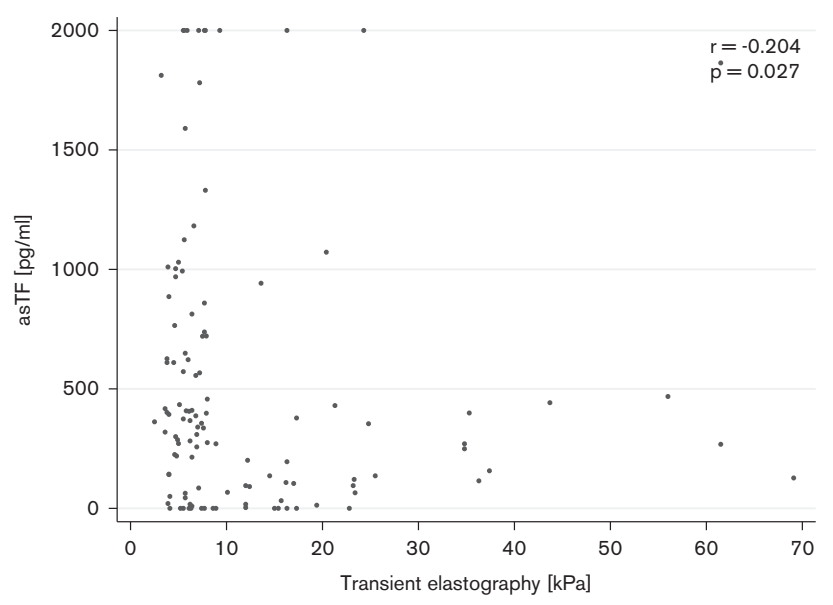

Fig. 3. Spearman's rank correlation analysis of circulating alternatively spliced tissue factor (asTF) levels and transient elastography in patients with grade $0-4$ fibrosis $(n=146)$. For illustration purposes, asTF values more than $2000 \mathrm{pg} / \mathrm{ml}$ are shown as $2000 \mathrm{pg} / \mathrm{ml}$.

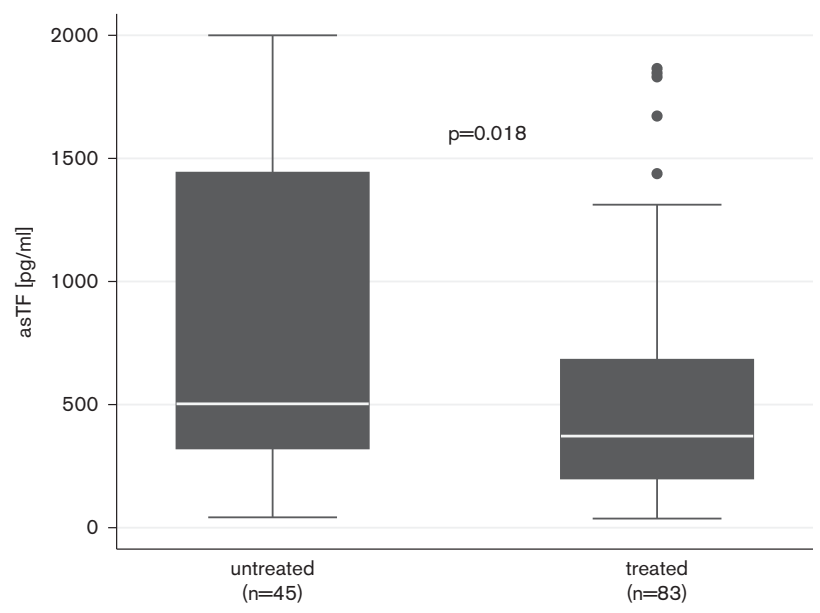

Fig. 4. Comparison of the distribution of plasma alternatively spliced tissue factor (asTF) levels in untreated patients with hepatocellular carcinoma (HCC) $(n=45)$ and treated patients with HCC $(n=83)$. Thick bars indicate median asTF concentrations, and thin bars the interquartile range. For illustration purposes, asTF values more than $2000 \mathrm{pg} / \mathrm{ml}$ are shown as $2000 \mathrm{pg} / \mathrm{ml}$.

fibrogenic process. Interestingly, it has been reported that the amount of TF was higher in patients with HIV, HCV, and HIV/HCV coinfection with advanced fibrosis than those with low fibrosis [29]. Indeed, Villa et al. [30] reported previously that an anticoagulant treatment with enoxaparin, which acts on microthrombosis, improves liver function and Child-Pugh score, thus decreasing the progression of liver disease.

In the context of this study, three important findings were revealed. First, treatment-naive patients with HCC had significantly higher asTF levels than those receiving treatment, indicating that liver cancer burden may favor production of circulating asTF. Second, patients with Child-Pugh A score had significantly lower asTF levels than those with Child-Pugh C score. Our data are in line with those reported by Rautou et al. [31] demonstrating that microparticle TF activity increases with cirrhosis severity as assessed by Child-Pugh score.

Third, patients with active liver disease had significantly higher circulating asTF levels than patients with no active

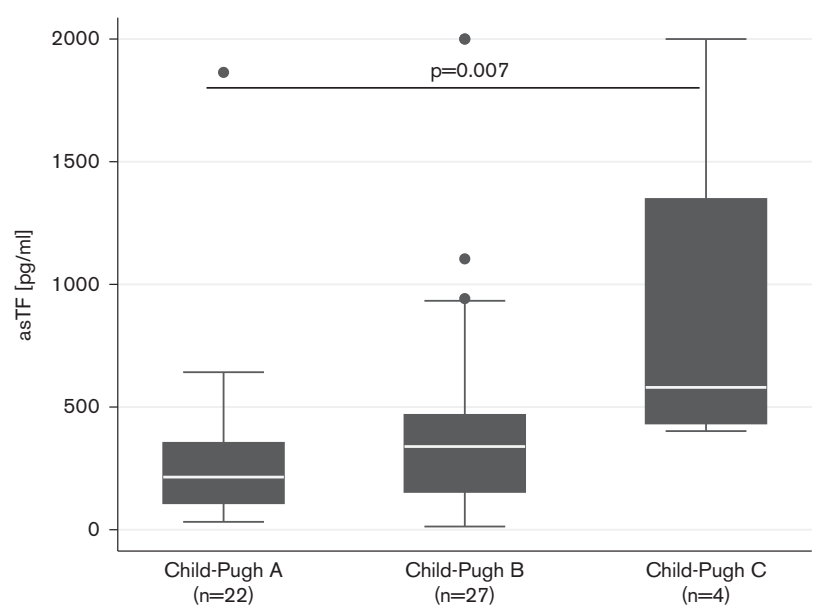

Fig. 5. Comparison of the distribution of plasma alternatively spliced tissue factor (asTF) levels in patients with cirrhosis with Child-Pugh A $(n=22)$, Child-Pugh B $(n=27)$ and Child-Pugh C $(n=4)$. Thick bars indicate median asTF concentrations, and thin bars the interquartile range. For illustration purposes, asTF values more than $2000 \mathrm{pg} / \mathrm{ml}$ are shown as $2000 \mathrm{pg} / \mathrm{ml}$.

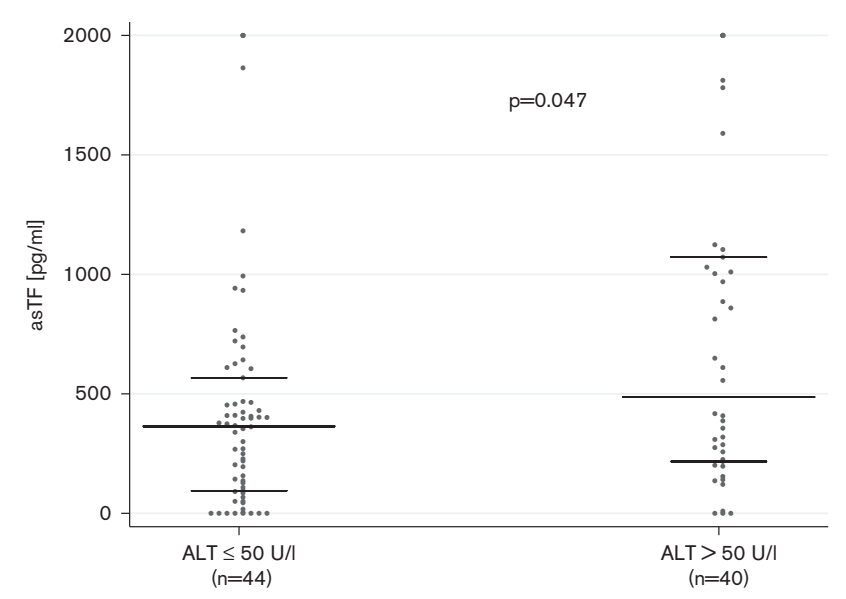

Fig. 6. Comparison of the distribution of plasma alternatively spliced tissue factor (asTF) levels in patients with no active liver disease [alanine aminotransferase $(\mathrm{ALT}) \leq 50 \mathrm{U} / \mathrm{I}](n=44)$ and active liver disease (ALT > 50 U/I) $(n=40)$. Thick bars indicate median asTF concentrations, and thin bars the interquartile range. For illustration purposes, asTF values more than $2000 \mathrm{pg} /$ $\mathrm{ml}$ are shown as $2000 \mathrm{pg} / \mathrm{ml}$.

liver disease. Therefore, we propose that the activity of liver disease affects the level of asTF in plasma. This could explain why F0-F1 patients (20 patients with active liver disease, $45.5 \%$ ) displayed higher asTF levels than patients with liver cirrhosis (11 patients with active liver disease, $16.9 \%)$.

Regarding the different liver cell types, hepatocytes express low levels of TF [5]. In mice, liver injury induces coagulation through upregulation of hepatocyte TF [32]. Kupffer cells and endothelial cells are able to express TF $[33,34]$. One interesting question that remains to be answered is whether there is an expression of flTF/asTF in hepatic stellate cells and if so, whether the expression levels differ in fibrosis compared with cirrhosis.

Our study has several limitations. It is a single-center study with a retrospective design. Furthermore, the sample sizes of some subgroups were small, so the study might not have had sufficient power to reveal statistically significant differences and/or associations. 


\section{Conclusion}

Our results showed for the first time that asTF plasma levels were elevated in patients with chronic liver disease. We found a correlation between plasma asTF and the degree of hepatic impairment, as assessed by Child-Pugh score. Importantly, we demonstrated that patients with HCC who have been treated displayed reduced as TF levels in comparison with untreated patients and that liver disease activity, defined as ALT more than $50 \mathrm{U} / \mathrm{I}$, influences asTF levels in plasma.

The pathogenic significance of our findings warrants further investigation.

\section{Acknowledgements}

This work was supported by the Swiss National Foundation for Scientific Research grants 310030_153436 and 314730_173127 (to A.A.S.). C.L. and V.Y.B. were partially supported by the NIH (grant R01CA190717 to V.Y.B.). M.M. has received a research grant "Young Talents in Clinical Research" (YTCR 14/17).

\section{Conflicts of interest}

There are no conflicts of interest.

\section{References}

1 Kuroe K, Kurokawa T, Nishikimi M, Nonami T, Harada A, Nakao A, et al. Effects of thromboxane A2 synthetase inhibitor on postischemic liver injury in rats. Eur Surg Res 199; 23:20-26.

2 Tripodi A, Salerno F, Chantarangkul V, Clerici M, Cazzaniga M, Primignani $\mathrm{M}$, et al. Evidence of normal thrombin generation in cirrhosis despite abnormal conventional coagulation tests. Hepatology 2005; 41:553-558.

3 Joshi N, Kopec AK, Ray JL, Cline-Fedewa H, Groeneveld DJ, Lisman T, et al. Von Willebrand factor deficiency reduces liver fibrosis in mice. Toxicol Appl Pharmacol 2017; 328:54-59.

4 Borkham-Kamphorst E, Zimmermann HW, Gassler N, Bissels U, Bosio A, Tacke F, et al. Factor VII activating protease (FSAP) exerts antiinflammatory and anti-fibrotic effects in liver fibrosis in mice and men. $J$ Hepatol 2013; 58:104-111.

5 Drake TA, Morrissey JH, Edgington TS. Selective cellular expression of tissue factor in human tissues. Implications for disorders of hemostasis and thrombosis. Am J Pathol 1989; 134:1087-1097.

6 Egorina EM, Sovershaev MA, Bjørkøy G, Gruber FXE, Olsen JO, Parhami-Seren B, et al. Intracellular and surface distribution of monocyte tissue factor: Application to intersubject variability. Arterioscler Thromb Vasc Biol 2005; 25:1493-1498.

7 Bogdanov VY, Balasubramanian V, Hathcock J, Vele O, Lieb M, Nemerson Y. Alternatively spliced human tissue factor: a circulating, soluble, thrombogenic protein. Nat Med 2003; 9:458-462.

8 Bogdanov $\mathrm{V}$. Blood coagulation and alternative pre-mrna splicing: an overview. Curr Mol Med 2006; 6:859-869.

9 Eisenreich A, Bogdanov VY, Zakrzewicz A, Pries A, Antoniak S, Poller W, et al. Cdc2-like kinases and DNA topoisomerase I regulate alternative splicing of tissue factor in human endothelial cells. Circ Res 2009; 104:589-599.

10 Chandradas S, Deikus G, Tardos JG, Bogdanov W. Antagonistic roles of four SR proteins in the biosynthesis of alternatively spliced tissue factor transcripts in monocytic cells. J Leukoc Biol 2010; 87:147-152.

11 Mackman N. Regulation of the tissue factor gene. Thromb Haemost 1997; 78:747-754.

12 Zumbach M, Hofmann M, Borcea V, Luther T, Kotzsch M, Müller M, et al. Tissue factor antigen is elevated in patients with microvascular complications of diabetes mellitus. Exp Clin Endocrinol Diabetes 1997; 105:206-212

13 Szotowski B, Goldin-Lang P, Antoniak S, Bogdanov WY, Pathirana D, Pauschinger $\mathrm{M}$, et al. Alterations in myocardial tissue factor expression and cellular localization in dilated cardiomyopathy. J Am Coll Cardiol 2005; 45:1081-1089.

14 Santucci RA, Erlich J, Labriola J, Wilson M, Kao KJ, Kickler TS, et al. Measurement of tissue factor activity in whole blood. Thromb Haemost 2000; 83:445-454.

15 Koyama T, Nishida K, Ohdama S, Sawada M, Murakami N, Hirosawa S, et al. Determination of plasma tissue factor antigen and its clinical significance. Br J Haematol 1994; 87:343-347.

16 Khorana A, Francis C, Menzies K, Wang JG, Hyrien O, Hathcock J, et al. Plasma tissue factor may be predictive of venous thromboembolism in pancreatic cancer. J Thromb Haemost 2010; 6:1983-1985.

$17 \mathrm{Pu} \mathrm{Y}$, Zhang S, Zhou R, Huang N, Li H, Wei W, et al. IL-17A upregulates expression of endothelial tissue factor in liver cirrhosis via the ROS/p38 signal pathway. Biochem Biophys Res Commun 2016; 470:41-47.

18 Rautou PE, Tatsumi K, Antoniak S, Owens AP, Sparkenbaugh E, Holle LA, et al. Hepatocyte tissue factor contributes to the hypercoagulable state in a mouse model of chronic liver injury. J Hepatol 2016; 64:53-59.

19 Van den Berg YW, van den Hengel LG, Myers HR, Ayachi O, Jordanova E, Ruf W, et al. Alternatively spliced tissue factor induces angiogenesis through integrin ligation. Proc Natl Acad Sci USA 2009; 106:19497-19502.

20 Unruh D, Turner K, Srinivasan R, Kocatürk B, Qi X, Chu Z, et al. Alternatively spliced tissue factor contributes to tumor spread and activation of coagulation in pancreatic ductal adenocarcinoma. Int $J$ Cancer 2014; 134:9-20.

21 Kocatürk B, van den Berg YW, Tieken C, Mieog JSD, de Kruijf EM, Engels CC, et al. Alternatively spliced tissue factor promotes breast cancer growth in a $\beta 1$ integrin-dependent manner. Proc Natl Acad Sci USA 2013; 110:11517-11522.

22 Unruh D, Sagin F, Adam M, van Dreden P, Woodhams BJ, Hart K, et al. Levels of alternatively spliced tissue factor in the plasma of patients with pancreatic cancer may help predict aggressive tumor phenotype. Ann Surg Oncol 2015; 22(S3):1206-1211.

23 Unruh D, Ünlü B, Lewis CS, Qi X, Chu Z, Sturm R, et al. Antibody-based targeting of alternatively spliced tissue factor: a new approach to impede the primary growth and spread of pancreatic ductal adenocarcinoma. Oncotarget 2016; 7:25264-25275.

24 Castera L, Yuen Chan HL, Arrese M, Afdhal N, Bedossa P, FriedrichRust M, et al. EASL-ALEH Clinical Practice Guidelines: noninvasive tests for evaluation of liver disease severity and prognosis. J Hepatol 2015; 63:237-264

25 Davila M, Robles-Carrillo L, Unruh D, Huo Q, Gardiner C, Sargent IL, et al. Microparticle association and heterogeneity of tumor-derived tissue factor in plasma: Is it important for coagulation activation? J Thromb Haemost 2014; 12:186-196.

26 Leonardi F, Maria N De, Villa E. Anticoagulation in cirrhosis: a new paradigm? Clin Mol Hepatol 2017; 23:13-21.

27 Mercer PF, Chambers RC. Coagulation and coagulation signalling in fibrosis. Biochim Biophys Acta 2013; 1832:1018-1027.

28 Zawaski S, Hammes M, Balasubramanian V. Alternatively spliced human tissue factor and thrombotic tendencies in hemodialysis patients. Int J Nephrol Urol 2010; 2:193-199.

29 Hodowanec AC, Lee RD, Brady KE, Gao W, Kincaid S, Plants J, et al. A matched cross-sectional study of the association between circulating tissue factor activity, immune activation and advanced liver fibrosis in hepatitis C infection. BMC Infect Dis 2015; 15:1.

30 Villa $\mathrm{E}$, Cammà $\mathrm{C}$, Marietta $\mathrm{M}$, Luongo $\mathrm{M}$, Critelli $\mathrm{R}$, Colopi $\mathrm{S}$, et al. Enoxaparin prevents portal vein thrombosis and liver decompensation in patients with advanced cirrhosis. Gastroenterology 2012; 143:1253-1260.

31 Rautou PE, Vion AC, Luyendyk JP, Mackman N. Circulating microparticle tissue factor activity Is increased in patients with cirrhosis. Hepatology 2014; 60:1793-1795.

32 Sullivan BP, Kopec AK, Joshi N, Cline H, Brown JA, Bishop SC, et al. Hepatocyte tissue factor activates the coagulation cascade in mice. Blood 2013; 121:1868-1874.

33 Ou ZB, Miao CM, Ye MX, Xing DP, He K, Li PZ, et al. Investigation for role of tissue factor and blood coagulation system in severe acute pancreatitis and associated liver injury. Biomed Pharmacother 2017; 85:380-388

34 Kim I, Oh JL, Ryu YS, So JN, Sessa WC, Walsh K, et al. Angiopoietin-1 negatively regulates expression and activity of tissue factor in endothelial cells. FASEB J 2002; 16:126-128. 